\title{
AUTO-MOBILE VEHICLE DIRECTION IN ROAD TRAFFIC USING ARTIFICIAL NEURAL NETWORKS
}

\author{
${ }^{1}$ B.SankaraSubramanian, ${ }^{2}$ R.Vasanth Kumar Mehta and ${ }^{3}$ N. Kumaran \\ ${ }^{1}$ Department of CSE, SCSVMV University, kanchipuram, Tamil Nadu, India \\ coolsankara@gmail.com, 919486118617 \\ ${ }^{2}$ Department of CSE, SCSVMV University, kanchipuram, Tamil Nadu, India \\ vasanthmehta@gmail.com, 919095984004 \\ ${ }^{3}$ Department of IT, SCSVMV University, kanchipuram, Tamil Nadu, India \\ natarajankumnerediffmaill. com, 919894744597
}

\begin{abstract}
So far Most of the current work on this area deals with traffic volume prediction during peak hours and the reasons behind accidents only. This work presents the analysis of automobile vehicle directing in various traffic flow conditions using Artificial neural network architecture. Now a days, due to unprecedented increase in automobile vehicular traffic especially in metroPolitian cities, it has become highly imperative that we must choose an optimum road route in accordance with our requirements. The requirements are : volume of the traffic, Distance between source and destination, no of signals in between the source and destination, the nature of the road condition, fuel consumption and Travel Timing. Artificial Neural networks, a soft computing technique, modeled after brain biological neuron functioning, helps to obtain the required road way or route as per the training given to it. Here we make use of Back propagation network, which changes the weights value of the hidden layers, thereby activation function value which fires the neuron to get the required output.
\end{abstract}

\section{KEYWORDS}

Vehicle route selection and direction, Artificial neural network, Road Traffic Analysis, Back

Propagation network.

\section{INTRODUCTION}

The daily mess that a commuter faces during his travel to office is felt a lot. People get stranded and become weird while passing through a heavy traffic condition. Though the Government plans and ideas like diverting the traffic during peak hours, change in timing between school-college goers and company workers, laying by-pass road and etc., to reduce the complexity in road traffic, it has not yielded a much expected result. As this is the case, the total burden lies on the shoulder of the commuters. They need some intelligent method by which they can avoid this

Sundarapandian et al. (Eds) : ACITY, AIAA, CNSA, DPPR, NeCoM, WeST, DMS, P2PTM, VLSI - 2013 pp. 49-55, 2013. (C) CS \& IT-CSCP 2013

DOI : $10.5121 /$ csit.2013.3406 
difficult condition and make their journey a pleasant one The main constraints felt by them are : traffic volume, no of signals they have to wait for clearance, road condition and fuel consumption, distance they have to travel if they divert their journey in various alternative routes to reach the destination. So, naturally, our aim is to select the best route that satisfies the user requirements during normal working days. But in some special days, like holidays or strike call, we can intuitively and easily judge the viable route to take up.

The paper is organized as follows. Section 1 is the Introduction. Section 2 deals with the basics of Artificial neural networks and back propagation network. Section 3 describes about the related work in this area. Section 4 describes the basic idea lying behind this work. and the architecture. Section 5 discusses about the performance evaluation and result details about performance and results. Section 6 finally concludes.

\section{ARTIFICIAL NEURAL NETWORK}

Artificial Neural Network [1] is a mimic of biological brain neuron for processing information given to it.The large number of neurons interconnected among themselves work altogether to solve a specific problem. The inputs given to it are classified into two types: Training data, which makes the network to learn to give the required output for a given set of inputs, Testing data, which tests the prediction accuracy of the network for randomly incoming data. It can be used for applications such as character and digit recognization or classification of data. It has got input layers , hidden layers and output layers. The function of the hidden layers is to fire an activation function like sigmoidal function to bring out the required output. The weight value, assigned between input and hidden layers changes themselves ,helps to minimize the error while getting the desired output.

\subsection{Back Propagation Network}

A single layer network can accomplish very limited number of tasks. Minsky and Papert(1969) [2] showed that a two-layer feed forward network can have many advantages but they question remained unanswered was " how the weights from the input to hidden layers can be adjusted?" The answer was given by Rumelhart, Hinton and Williams in 1986. They reasoned that by propagating errors of the output layers back into the units of the hidden layers, we can adjust the weight values thereby making the network more adaptive and accurate. This method is also called "Back Propagation Learning Rule". It is a generalization of delta rules for non-linear activation functions and multi-layer networks and a systematic method of training the networks.

A Back propagation network[2] consists of at least three layers of units:

1. An input layer

2. At least one intermediate layer called hidden layer.

3. An output layer.

The units are connected in feed-forward fashion with input units are fully connected to the hidden layers and hidden layers are fully connected to the output layers. With back propagation networks, learning occurs during learning phase. The patterns are applied to the input units and propagated forward. The output layer pattern of activation is compared with the expected Pattern to calculate the error signal. The error signal at the output pattern is then propagated back to the input units in order to appropriately adjust the weights in each layer of the network. Once the 
back propagation network has learnt to classify correctly for a set of inputs, a second set of inputs can be given to see how well it classify the untrained inputs. The important point is to see how well the network generalizes.

\section{THE RELATED WORK}

a) Konjicija.S, Avdagic . Z and Meier.G, Wurmthaler.C in their paper [4] have shown a simple ability of using neural networks in longitudinal vehicle guidance. Here neural networks learn from acquired real driver data, and also the driver behaviour styles for both ends ie., extremely comfort to extremely sportive ones. This scenario is shown as a simulated model based on longitudinal trajectory generation. In this an adjustable comfort parameter is used for different sorts of driver behaviour.

b) In their paper Lioing Fua and L.R.Rilettba [5] presents an artificial neural network (ANN) based method for estimating route travel times between individual locations in an urban traffic network.. The methodology developed in this paper assumes that route travel times are timedependent and stochastic and their means and standard deviations need to be estimated. Three feed-forward neural networks are developed to model the travel time behaviour during different time periods of the day the AM peak, the PM peak, and the off-peak. These models are subsequently trained and tested using data simulated on the road network for the City of Edmonton, Alberta. The ANN model is compared with a distance-based model and a shortest path algorithm. The practical implication of the ANN method is subsequently demonstrated within a dial-a- ride para-transit vehicle routing and scheduling problem. The computational results show that the ANN-based route travel time estimation model is appropriate, with respect to accuracy and speed, for use in real applications.

c) Baluja, S presents [6] creation of an artificial neural network based autonomous land vehicle controller. The performance of evolved controllers is better in unseen situations than those trained with an error back propagation learning algorithm . An overview of the previous connectionist based approaches is described and the exploration of methods for reducing the high computational costs of training ANN with evolutionary algorithms is done. The evolutionary algorithms guided by error metrics shows improved performance over those guided by the standard sum-squared error metric. Both g evolutionary search and error back propagation are integrated in this work.

d) In his paper, Dean A,Pomelleau [7] in The ALVINN (Autonomous Land Vehicle In a Neural Network) project describes about real-time training of ANN to perform difficult perception tasks. It is a back propagation network modeled to drive the CMU Navlab, a modified Chevy van. He describes the training techniques for ALVINN to learn in under 5 minutes to autonomously control the Navlab by observing the human driver reactions $r$. This enables the ALVINN to drive in a variety of conditions including single-lane paved and unpaved roads, and multilane lined and unlined roads, at speeds of up to 20 miles per hour.

\section{THE PROPOSED WORK}

We take source to destination distance (categorized as Lengthy, Medium and Short),Volume of Traffic(categorized as Peak or Heavy, Medium and Mild), No of Signals( categorized as High, Medium and Low), Road Condition(Categorized as Smooth, Good and Bad), Travel 
Timing(Categorized as High, Normal and Low) as input parameters as shown in fig 2(Neural Network Back Propagation Architecture) We eliminate fuel consumption just because it is indicated by the source and destination distance and Travel Timing selection categories. So there are five input parameters and each have three categories, thus giving a total of 243(35) combinations. Here we consider 5 alternative routes as the output nodes. They are :

S-X1-Y1-Z1-D,

S-W2-X2-Y2-Z2,

S-X3-Y3-Z3-D,

S-V4-W4-X4-Y4-Z4-D,

S-X5-Y5-Z5-D

where $\mathrm{V}, \mathrm{W}, \mathrm{X}, \mathrm{Y}$ and $\mathrm{Z}$ intermediate nodes along the routes.

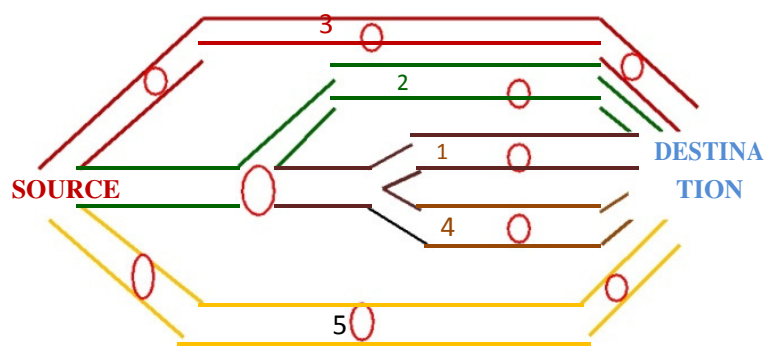

Fig.1 Main Route with Alternatives

1

$2,3,4,5$
Main route Alternative routes

Signal point

So there are 15 input nodes and 5 output nodes. The hidden layers nodes are fixed as 10 , the mean value of input and output nodes as per thumb rule. The Sigmoid function is selected as the activation function in the hidden layer. Each input node is assigned a weight and fully connected to the hidden layer and in turn hidden layer nodes are again fully connected to output nodes. Out of total 243 combinations, we take any 180 as the training data sets. These inputs are uses to train the network to produce the actual result.

For example, if we choose

Source to Destination distance as Medium $(0,1,0)$,

Volume of Traffic as Mild $(0,0,1)$

No of Signals as Medium $(0,1,0)$

Road Condition as $\operatorname{Smooth}(1,0,0)$

Travel Timing as $\operatorname{Low}(0,0,1)$

Then network is trained, say, to choose Route 5 (the route with higher output layer value) Similarly for other input conditions the network is trained and we obtain Route information as 
follows (only few samples are shown)

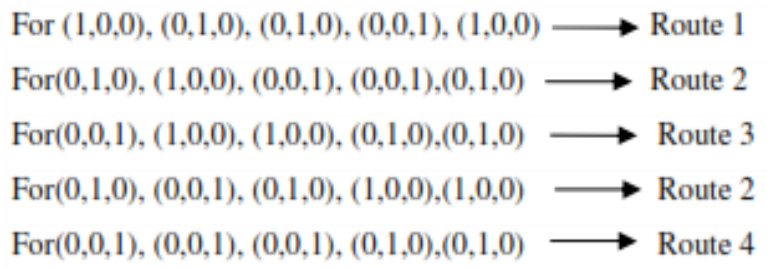

Now the testing data is fed into the network from unforeseen inputs. The error value, if any, in output layer is fed back by the back propagation network and weight values of hidden layers are changed accordingly to get the desired output. This is the usual practice for any working day/Normal day. But for holidays or strike call we can choose always the shortest route(Default route) to reach the destination.

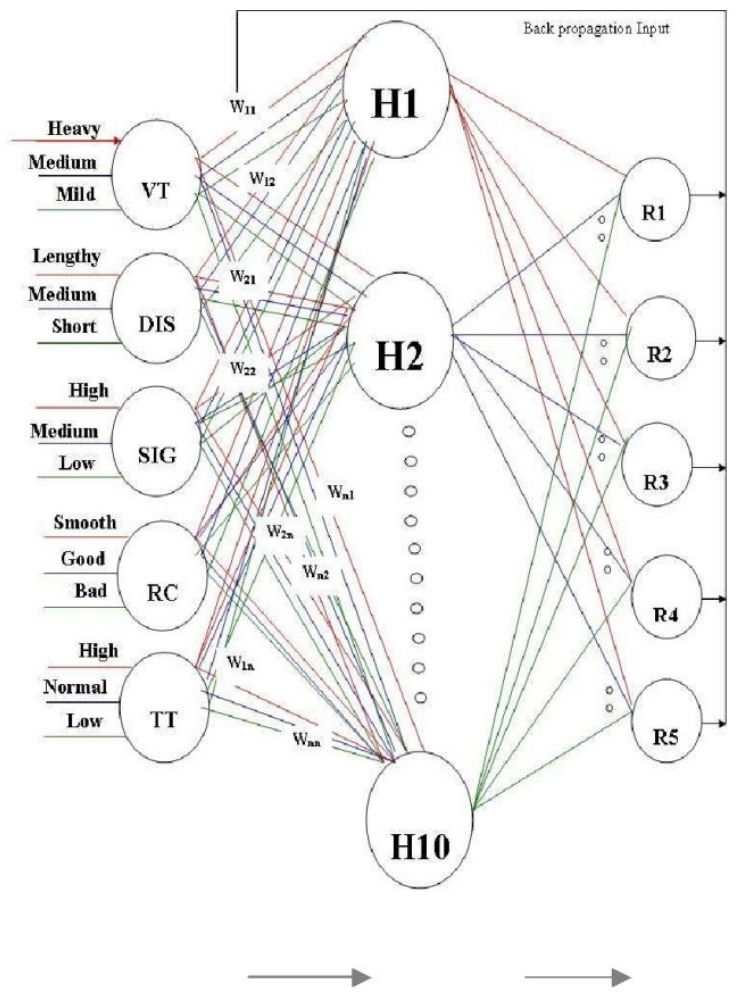

Figure.2 Neural Network Back Propagation Architecture

VT - Volume of Traffic

DIS - Distance between source and destination

SIG -- No of Signals

$\mathrm{RC}$-- Road Condition

TT - Travel Timing

$\mathrm{H} 1, \mathrm{H} 2, \ldots . . \mathrm{H} 10$ - Hidden layer Units

R3 -- Main (Default) Route 
R1, R2, R4, R5 - Alternative routes

\section{PERformance Evaluation AND ReSult}

We considered the Koyambedu-Broadway route in Chennai,TamilNadu, India as the source and destination pair and we put one route as the normal and selected other 4 routes(left-side 2 and right-side 2 to the normal route,see fig 1) through various road links. Initially we tested manually about the outcome of the best route among the five alternative route chosen for various input parameters and these outcomes are used to train the network for various combinations of input conditions. Then the testing data are given and the results obtained are summarized as follows

Table 1, Percentage Accuracy Of Selection And Rmse

\begin{tabular}{|c|c|c|c|}
\hline Parameters & $\begin{array}{l}\text { Network } \\
\text { tested } \\
\text { manually }\end{array}$ & $\begin{array}{c}\text { Network tested } \\
\text { without Back- } \\
\text { Propagation }\end{array}$ & $\begin{array}{c}\text { Network tested } \\
\text { with Back- } \\
\text { Propagation }\end{array}$ \\
\hline $\begin{array}{c}\text { Accuracy } \\
\text { of Selection }\end{array}$ & $95.40 \%$ & $84.90 \%$ & $91.20 \%$ \\
\hline $\begin{array}{c}\text { Root-Mean } \\
\text { Square } \\
\text { Error }\end{array}$ & 0.28 & 0.42 & 0.12 \\
\hline
\end{tabular}

Equation : RMS for a Neural Network [3]

$$
x_{r m s}=\sqrt{\frac{1}{n} \sum_{i=1}^{n}\left(\text { actual }_{i}-\text { ideal }_{i}\right)^{2}}
$$

So we can conclude that the network with back propagation performs better than without it. The reason behind the higher error value in case of without back propagation network is the curse of dimensionality. Here there are 15 inputs and the categorical division(each 3) of it results in 243 combinations. The no of inputs can be reduced if we consider ANY CONDITION, say, Road condition ANY or No of Signals ANY Numbers. Then the input reduces to $27\left(3^{3}\right)$ from $243\left(3^{5}\right)$ and the network performs more efficiently than with higher inputs.

\section{CONCLUSION}

This paper presents the novel approach of neural network based analysis of best route selection for Automobile vehicle travel and is the first of its kind. There are certain assumptions like : The road condition remain same independent of time, the no of alternate routes remain fixed ,no of signals are fixed, Volume of traffic remain same throughout the route during the time period considered and the regulation over the movement of different vehicle types such as Heavy, Medium and Light. But these assumptions are changeable and dynamic in nature. Hence the results obtained may not hold good and give only approximate measures. In Future work, we are proposing to analyze these dynamic nature of traffic flow conditions. 


\section{ACKNOWLEDGEMENT}

We sincerely thank, University management, for giving constant support and Mr.L.Ganesh for his valuable help in publishing this manuscript.

\section{REFERENCES}

[1] www.doc.ic.ac.uk/ nd/surprise_96/journal/vol4/cs11/ report.html :

[2] http://www.myreaders.info/03_Back_Propagation_Network.pdf

[3] www.heatonresearch.com > ... > Chapter 4: How a Mac hine Learns

[4] Konjicija, S.; Avdagic,Z.; Meier,G.; Wurmthaler, "Longitudinal vehicle guidance using neural networks Computational Intelligence in Robotics and Automation”,. CIRA 2005.Proceedings.2005 IEEE International symposium.Page(s): $685-688$

[5] "Estimation of Time-Dependent, Stochastic Route Travel Times Using Artificial Neural Networks". LIPING FUa'*and L.R.RILETTba 1Department of Civil Engineering,University of Waterloo, Canada,; 2Department of Civil Engineering and Texas Transportation Institute, Texas A\&M University, Texas, , USA

[6] Baluja, S.Sch. "Evolution of an artificial neural network based autonomous land vehicle controller Systems, Man, and Cybernetics", Part B: Cybernetics, IEEE Transactions on Date of Publication: June 1996 Author(s): of Comput. Sci., Carnegie Mellon Univ., Pittsburgh, PA Volume: 26 , Issue: 3Page(s): $450-463$

[7] Dean A. Pomerleau, "Efficient training of artificial neural networks for autonomous navigation" School of Computer Science, Carnegie Mellon University, Pittsburgh, PA 15213 USA, Neural Computation journal Volume 3 Issue 1, Spring 1991 Pages 88-97,MIT Press Cambridge, MA, US

\section{Authors}

B.Sankara Subramanian. B.E(EEE), M.E(CSE), (Ph.D) He was born and brought-up in Madurai, a small town in southern Peninsular India famous for temples. He graduated B.E(EEE) in First Class from Madurai Kamaraj University in 1997 and his Post Graduate M.E(CSE) in 2007 from Anna University, Chennai. He has around 10 years of teaching experience in Electrical and Computer Science Departments. His area of interest includes Information and Database Systems, Data warehousing and Data mining, Algorithms, Fuzzy Logic, Rough set Theory and Knowledge Retrieval. He is currently doing his Ph.d in Soft Computing area in SCSVMV University, Kanchipuram where he is presently employed.

R.Vasanth Kumar Mehta. M.S., (Ph.d), He completed his graduate studies from BITS, Pilani and is presently pursuing Ph.D. from Sri Chandrasekharendra Saraswathi Viswa Mahavidyalaya(SCSVMV University), Kanchipuram, where he is working as Assistant Professor in the Department of Computer Science and Engineering. Areas of interest include Data Mining and Highperformance Computing

N.Kumaran did B.E(CSE),M.Tech(IT), (Ph.D). He did his B.E(Computer science and Engineering) in CIT, Coimbatore, Tamil Nadu, India in the year 1998. And M.Tech(IT) Sathyabama University, Chennai in 2007. Currently he is pursing Ph.D(Computer networks) in NIT, Trichy. His areas of interest include Computer Networks, Neural networks, Network Security and cryptography. He has got around12 years of teaching experience various engineering colleges.
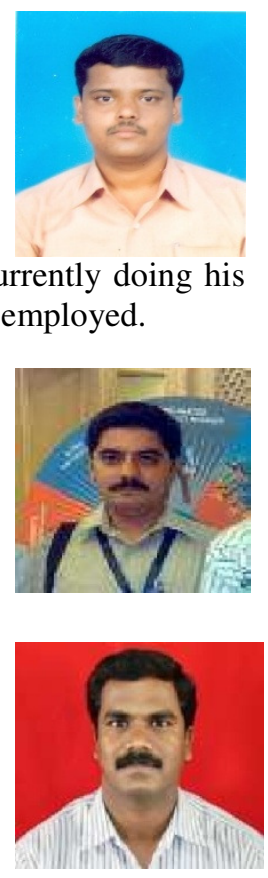